\title{
Virtual Reality for Mineral Surface Design
}

\author{
NILS SCHEWE $^{1}$, AND PETER THISSEN $^{1 *}$ \\ ${ }^{1}$ Institute of Functional Interfaces, Karlsruhe Institute of Technology, 76344 Eggenstein-Leopoldshafen, Germany \\ (nils.schewe@kit.edu and *correspondence: peter.thissen@kit.edu)
}

\section{Using VR as integrated workflow}

Reactions between mineral structures and surrounding media happen at interfaces. Understanding these interfaces is mandatory for the development of functional materials. Modeling mineral interfaces at the atomic level is complex and time-consuming, since 3D objects have to be created using 2D workflows [1]. To ease up the creation of complex interfaces and their environment, we employ a newly constructed virtual reality (VR) laboratory [2]. The connection of this virtual work space with both experimental data and high preformance computation (HPC) via direct implementation allows not only for the visualization [3], but also the active manipulation of sophisticated materials. As prominent example we show the optimization of gas adsorption on a calcite surface through placing $\mathrm{CO}_{2}$ molecules by hand and subsequent electronic energy minimalization of the resulting structure via Vienna Ab initio Simulation Package (VASP) on a HPC-cluster. The VR-laboratory acts both as the workspace and the graphical user interface (GUI) for the implemented HPC-applications. Setting the initial position and orientation of the small molecule relative to the surface by hand gives a new degree of freedom and ensures that the scientist can focus on the subject instead of handling software.

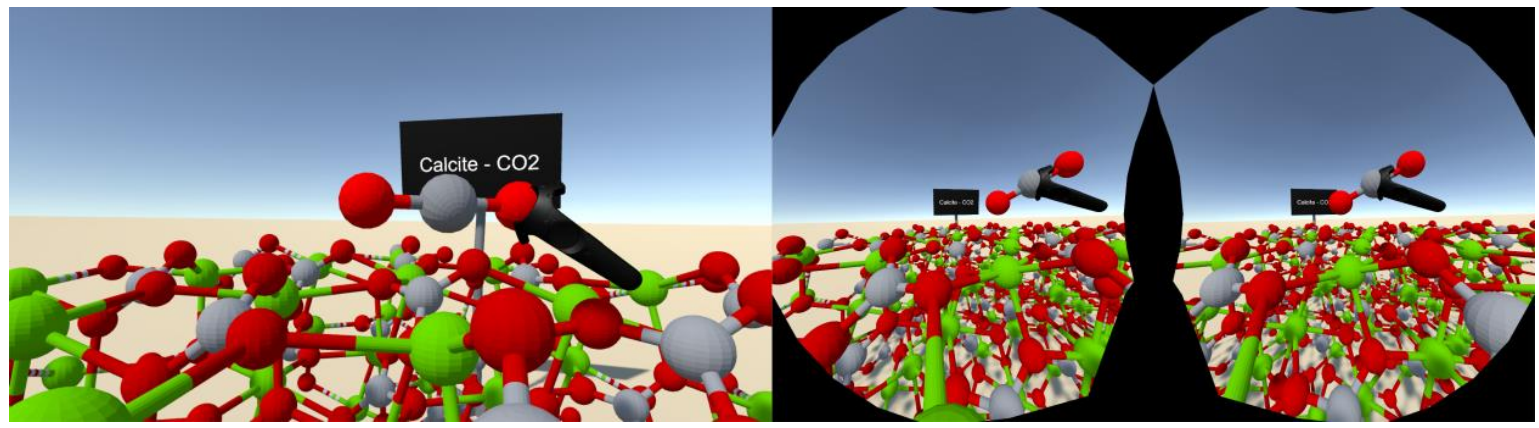

Figure 1: The VR-laboratory lets the scientist try various orientations and positions of the $\mathrm{CO}_{2}$ on the calcite slab in a very fast and intuitive way.

The implementation of additional functionalities like a virtual lab book will help to accellerate the workflow significantly. Background databases, to be established until the end of the year, will provide a large amount of chemical structures and material information to further enhance the variability of tasks that can be worked on in the VR-laboratory.

[1] Thissen et al. (2014), J. Phys. Chem. C, 118, 8007-8013.

[2] Honysz \& Dobrzański (2017), Journal of Achievements in Materials and Manufacturing Engineering, 84/2, 76-84.

[3] Hagita et al. (2019), ACS Omega, 4, 2, 3990-3999. 